\title{
Internal Energy Based Grid-Forming Control for MMC-HVDC Systems with Wind Farm Integration
}

\author{
Renxin Yang \\ Key Laboratory of Control of Power \\ Transmission and Conversion \\ Shanghai Jiao Tong University \\ Shanghai, China \\ frank_yang@sjtu.edu.cn \\ Chen Zhang \\ Department of Electrical Engineering \\ University of Denmark \\ Copenhagen, Denmark \\ chezh@elektro.dtu.dk
}

\author{
Gang Shi \\ Key Laboratory of Control of Power \\ Transmission and Conversion \\ Shanghai Jiao Tong University \\ Shanghai, China \\ gangshi@sjtu.edu.cn \\ Gen Li \\ School of Engineering \\ Cardiff University \\ Cardiff, U.K. \\ lig9@cardiff.ac.uk
}

\author{
Xu Cai \\ Key Laboratory of Control of Power \\ Transmission and Conversion \\ Shanghai Jiao Tong University \\ Shanghai, China \\ xucai@sjtu.edu.cn
}

\begin{abstract}
The virtual synchronous control is regarded as an effective solution for grid-tied converters to operate under weak grid conditions and provide inertia support. However, for those grid-tied converters which control the DC voltage, e.g., the receiving end converter (REC) in modular multilevel converter based high-voltage direct-current (MMC-HVDC) transmission systems with wind farm integration, the application of virtual synchronous control is difficult due to the cascade control loops. The dynamic performance of DC voltage control may also be degraded. To address these issues, a novel grid-forming control strategy with real-time inertia support and fast DC voltage control is proposed for the REC. The cascade control loops of the virtual synchronous control are simplified by utilizing the internal energy stored in sub-module capacitors to emulate synchronous generator rotors. Moreover, the DC voltage is decoupled with the submodule capacitors voltage, thus being robust to sudden power change. Simulation results in PSCAD/EMTDC show that the proposed control realizes fast inertia support and desired dynamic control of the DC voltage, especially in weak grid operation.
\end{abstract}

Keywords-VSC-HVDC, MMC, wind farm, frequency support, grid-forming control

\section{INTRODUCTION}

Modular multilevel converter-based high-voltage directcurrent (MMC-HVDC) transmission system[1] is regarded as an alternative solution for integrating large-scale offshore wind farms (OWFs) compared to its HVAC counterpart which has the capacitive charging effect of HVAC submarine cables. However, the replacement of synchronous power sources by MMC-HVDC system with OWF integration will reduce the inertia of the local power grid, which may lead to frequency stability issues. Moreover, the weak grid with a large penetration of inverter-based power sources may not be able to provide a stiff voltage at the point of common coupling. In this case, the conventional grid-following control strategy, which utilizes the phase-locked loop (PLL) to align the current reference of the vector current control, may suffer from oscillation issues if the parameters of PLL are not properly designed.

The grid-forming control is an effective solution for gridtied converters to operate under weak grid conditions and provide inertia support. Different synchronization methods

This work is supported by the National Key R\&D Plan of China (Grant Number: 2018YFB1501300), and the Natural Science Foundation of Shanghai (Grant Number: 20ZR1427100) have been designed to replace the PLL [1]-[5]. However, most of the grid-forming control strategies only include the active power loop, and the DC side is simplified as a constant voltage source. For the receiving end converter (REC) in the MMCHVDC system with OWF integration, an additional $U_{\mathrm{dc}}-P$ loop is needed to regulate the DC voltage. Such a multiloops control structure poses a great challenge in tuning the parameters.

In order to simplify the converter control loops, the inherent dynamics of the DC side capacitor can be used to emulate the rotor of the synchronous generator (SG) [6]. In [7], a lead-lag transfer function is proposed in the converter control system to enhance the damping capability. In [8], the amplitude of the output $\mathrm{AC}$ voltage is regulated to perform the damping capability. These methods are mostly designed for 2level voltage source converters (VSCs). A common shortcoming of them is that the control of DC voltage is coupled with the grid-synchronization process. The DC voltage may be sensitive to sudden power change from the sending end, and cannot be controlled rapidly and precisely. Therefore, these control strategies cannot be easily extended to multi-terminal HVDC systems, in which the power flow may change frequently. In addition, when the DC voltage is utilized to deliver grid frequency information and facilitate OWFs to provide communication-less inertia support [8], this feature will influence the accuracy of grid frequency information and cause unexpected inertia response.

In this letter, a novel control strategy for the REC in MMC-HVDC system with OWF integration is proposed. The proposed control consists of two parts. At the AC side, the overall stored energy of sub-module (SM) capacitors is calculated and used to emulate the SG's rotor dynamics. This part will achieve the grid-synchronization of the REC without using a PLL, meanwhile the total energy of SM capacitors can remain balanced. At the $\mathrm{DC}$ side, the $\mathrm{DC}$ voltage is controlled to track grid frequency variation, thus delivering the grid frequency information to the SEC. In addition, the decoupled DC voltage control method is proposed and compared to the conventional DC voltage control method in this part. The results show that the decoupled DC voltage control method has a better dynamic performance of DC voltage regulation than the conventional method, as well as a higher accuracy when delivering grid frequency information. Thanks to this feature, the SEC can help the OWFs to sense grid frequency variation and provide communication-less inertia support to the onshore grid. 


\section{THE AC SIDE CONTROL: AUTONOMOUS- SYNCHRONIZING CONTROL OF RECS}

As shown in Eq. (1), the total stored energy $W_{\text {total }}$ in SM capacitors of the MMC can be calculated by:

$$
W_{\text {total }}=\sum_{n=1}^{6 N} \frac{1}{2} C_{\mathrm{SM}} U_{\mathrm{SMn}}^{2},
$$

where $C_{\mathrm{SM}}$ is the SM capacitance and $U_{\mathrm{SMn}}$ is the SM voltage.

In order to emulate the swing equation of the SG, a link between $W_{\text {total }}$ and $\omega_{\text {rec }}$ is established:

$$
\left\{\begin{array}{l}
\Delta \bar{\omega}_{\text {rec }}=\frac{\Delta \bar{W}_{\text {total }}}{2 H_{\mathrm{v}}} \\
\bar{W}_{\text {total }}=\frac{W_{\text {total }}}{P_{\text {ac_nom }}}
\end{array},\right.
$$

where $H_{\mathrm{v}}$ is the virtual inertia.

The dynamic response of the $W_{\text {total }}$ to input and output active power is:

$$
\bar{P}_{\mathrm{dc}}-\bar{P}_{\mathrm{ac}}=\frac{\mathrm{d} \Delta \bar{W}_{\text {total }}}{\mathrm{d} t}=2 H_{\mathrm{v}} \frac{\mathrm{d} \Delta \bar{\omega}_{\text {rec }}}{\mathrm{d} t}
$$

$P_{\mathrm{dc}}$ is the DC side input active power of REC, and $P_{\mathrm{ac}}$ is the AC side output active power of REC. It should be noted that the internal energy of SM capacitors is only used for gridsynchronization. The inertia that the total SM capacitors can provide is very limited. Due to this physical constraint, the virtual inertia coefficient $H_{\mathrm{v}}$ should not be much larger than the equivalent time constant of all SM capacitors, which is usually in the range: 30 50 ms.

Moreover, the damping capability can be achieved by regulating the $\mathrm{AC}$ voltage:

$$
\Delta \bar{U}_{\mathrm{ac}}=\frac{\Delta \bar{W}_{\text {total }}}{2 H_{\mathrm{v}} \bar{P}_{\mathrm{ac} 0}} D_{\mathrm{v}},
$$

where $D_{\mathrm{v}}$ is the damping coefficient.

The change of $\mathrm{AC}$ voltage will lead to the change of REC's output AC power $P_{\text {ac }}$ :

$$
\begin{aligned}
\bar{P}_{\text {ac } \_ \text {new }} & =\bar{P}_{\text {ac }}+\Delta \bar{P}_{\text {ac }}=\bar{P}_{\text {ac }}+\frac{P_{\text {ac } 0}}{P_{\text {ac_nom }}} \frac{\Delta U_{\text {ac }}}{U_{\text {ac_nom }}} . \\
& =\bar{P}_{\text {ac }}+\frac{\Delta \bar{W}_{\text {total }}}{H_{\mathrm{v}}} D_{\mathrm{v}}=\bar{P}_{\text {ac }}+D_{\mathrm{v}} \Delta \bar{\omega}_{\text {rec }}
\end{aligned}
$$

Using $P_{\text {ac new }}$ to replace $P_{\text {ac }}$ in Eq. (3), the output equation of the REC can be written as:

$$
\bar{P}_{\mathrm{dc}}-\left(\bar{P}_{\mathrm{ac}}+D_{\mathrm{v}} \Delta \bar{\omega}_{\mathrm{rec}}\right)=\frac{\mathrm{d} \Delta \bar{W}_{\text {total }}}{\mathrm{d} t}=2 H_{\mathrm{v}} \frac{\mathrm{d} \Delta \bar{\omega}_{\text {rec }}}{\mathrm{d} t} .
$$

Therefore:

$$
\bar{P}_{\mathrm{dc}}-\bar{P}_{\mathrm{ac}}=2 H_{\mathrm{v}} \frac{\mathrm{d} \Delta \bar{\omega}_{\mathrm{rec}}}{\mathrm{d} t}+D_{\mathrm{v}} \Delta \bar{\omega}_{\mathrm{rec}} .
$$

The Eq. (7) of the REC performs like the swing equation of the SG. Therefore, the REC will achieve the selfsynchronizing characteristic like an SG, i.e., the following correlations can be established:

$$
\omega_{\mathrm{g}} \uparrow \Rightarrow \delta \downarrow \Rightarrow P_{\mathrm{ac}} \downarrow \Rightarrow W_{\text {total }} \uparrow \Rightarrow \omega_{\text {rec }} \uparrow .
$$

The diagram of this internal energy based autonomous synchronizing control is shown in Fig. 1.

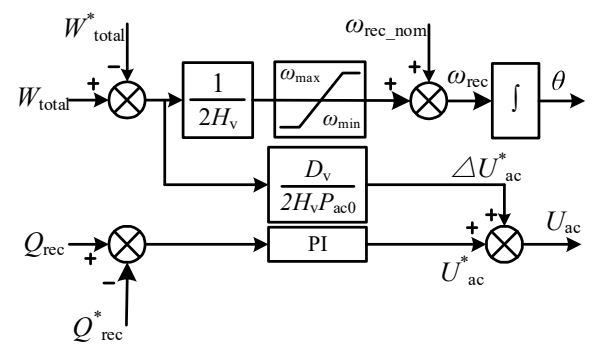

Fig. 1. Internal energy based autonomous synchronizing control of REC.

The output active power of the REC is:

$$
\left\{\begin{array}{l}
\bar{P}_{\mathrm{ac}}=\frac{\bar{U}_{\mathrm{rec}} \bar{U}_{\mathrm{g}}}{\bar{X}_{\Sigma}} \delta \\
\delta=\int\left(\omega_{\mathrm{rec}}-\omega_{\mathrm{g}}\right) d t
\end{array} .\right.
$$

Considering $P_{\mathrm{dc}}$ and $P_{\mathrm{ac}}$ as constants. the transfer function between $\omega_{\mathrm{g}}$ and $\omega_{\text {rec }}$ can be derived from Eq. (7) and Eq. (9):

$$
\Delta \bar{\omega}_{\text {rec }}=\frac{\Delta \bar{W}_{\text {total }}}{2 H_{\mathrm{v}}}=G(s) \Delta \bar{\omega}_{\mathrm{g}}=\frac{\omega_{0}}{2 H_{\mathrm{v}} \bar{X}_{\Sigma} s^{2}+\bar{X}_{\Sigma} D_{\mathrm{v}} s+\omega_{0}} \Delta \bar{\omega}_{\mathrm{g}} .
$$

Taking the parameters in Table I for an example. The short-circuit-ratio (SCR) is set to 2.5, i.e., the equivalent grid impedance $\bar{X}_{\Sigma}$ is 0.4 p.u. $H_{\mathrm{v}}$ and $D_{\mathrm{v}}$ are set to $0.05 \mathrm{~s}$ and 13 , respectively. It can be calculated that the cut-off frequency of $G(\mathrm{~s})$ is $85 \mathrm{rad} / \mathrm{s}$, which is much larger than the speed of grid frequency variation (usually $6 \mathrm{rad} / \mathrm{s}$ ). Therefore, $\omega_{\text {rec }}$ can keep synchronized with $\omega_{\mathrm{g}}$ in approximately real time.

TABLE I

PARAMETERS OF THE HVDC SYSTEM

\begin{tabular}{cc}
\hline \hline Parameters & Values \\
\hline Rated DC voltage & $\pm 320 \mathrm{kV}$ \\
Rated AC voltage (line-to-line) & $333.13 \mathrm{kV}$ \\
Transformer leakage inductance & $0.14 \mathrm{p.u}$. \\
Rated active power & $1000 \mathrm{MW}$ \\
\hline REC and SEC & \\
\hline SM number & 412 \\
Redundant SMs & 31 \\
SM capacitance & $11 \mathrm{mF}$ \\
Arm inductance & $80 \mathrm{mH}$ \\
\hline
\end{tabular}

\section{ThE DC SIDE CONTROL: DC Voltage CONTROL OF RECS}

Two different methods can be utilized to control the DC voltage: the conventional DC voltage control and the decoupled DC voltage control[10].

\section{A. Conventional DC Voltage Control of REC}

In the conventional DC voltage control method, the DClink voltage of the MMC is usually controlled by the AC side active power, which is similar to the two-level VSC. The sum of the inserted submodules of the upper and lower arms is a constant $N$. Therefore, the DC-link voltage $U_{\mathrm{dc}}$ is coupled with the average submodule capacitor voltage $U_{\text {SMav }}$ :

$$
\bar{U}_{\mathrm{dc}}=\frac{N U_{\mathrm{SMav}}}{U_{\mathrm{dc} \_ \text {nom }}} .
$$

The internal energy of SM capacitors can be written as:

$$
\left\{\begin{array}{l}
\Delta \bar{W}_{\text {total }}=\Delta\left(\frac{6 N C_{\mathrm{SM}} U_{\mathrm{SMav}}^{2}}{2 P_{\text {nom }}}\right)=2 T_{\mathrm{MMC}} \frac{\Delta U_{\text {SMav }}}{U_{\text {SMav_nom }}} . \\
T_{\mathrm{MMC}}=\frac{6 N C_{\mathrm{SM}} U_{\mathrm{SMav} \_n o m}^{2}}{2 P_{\text {nom }}}
\end{array}\right.
$$


$C_{\mathrm{SM}}$ is the submodule capacitance. $T_{\mathrm{MMC}}$ is the time constant of MMC submodules, which is usually $30-50 \mathrm{~ms}$ in practical project.

It can be observed from Eq. (10), (11) and (12) that:

$$
\begin{aligned}
\Delta \bar{U}_{\mathrm{dc}} & =\frac{N \Delta U_{\mathrm{SMav}}}{U_{\mathrm{dc} \_ \text {nom }}}=\frac{\Delta \bar{W}_{\text {total }}}{2 T_{\mathrm{MMC}}} \frac{N U_{\text {SMav_nom }}}{U_{\text {dc_nom }}} . \\
& =\frac{\Delta \bar{W}_{\text {total }}}{2 T_{\mathrm{MMC}}}=\frac{H_{\mathrm{v}} G(s)}{T_{\mathrm{MMC}}} \Delta \bar{\omega}_{\mathrm{g}} .
\end{aligned}
$$

Therefore, the DC voltage can keep synchronized with the grid frequency autonomously. Since the grid frequency variation is very small, the DC voltage can be controlled within the acceptable range. Moreover, the grid frequency variation is reflected on DC voltage according to Eq. (13), and the SEC can be informed with the grid frequency information without communication. Considering that the grid frequency variation is usually less than $\pm 1 \%$, and the DC voltage deviation is usually less than $\pm 5 \%, H_{\mathrm{v}} / T_{\mathrm{MMC}}$ should be less than 5 .

However, the DC voltage is coupled with the output frequency $\omega_{\text {rec }}$ and the internal energy $W_{\text {total }}$ of the REC with the conventional control strategy. The transfer function between $U_{\mathrm{dc}}$ and $P_{\mathrm{dc}}$, and between $U_{\mathrm{dc}}$ and $U_{\mathrm{g}}$ can be derived from Eq. (7):

$$
\begin{aligned}
\Delta \bar{U}_{\mathrm{dc}} & =\frac{\Delta \bar{W}_{\text {total }}}{2 T_{\mathrm{MMC}}}=\frac{H_{\mathrm{v}}}{T_{\mathrm{MMC}}} \Delta \bar{\omega}_{\mathrm{rec}} \\
& =\frac{H_{\mathrm{v}} \bar{X}_{\Sigma} s}{T_{\mathrm{MMC}}\left(2 H_{\mathrm{v}} \bar{X}_{\Sigma} s^{2}+\bar{X}_{\Sigma} D_{\mathrm{v}} s+\omega_{0}\right)} \Delta \bar{P}_{\mathrm{dc}} \\
\Delta \bar{U}_{\mathrm{dc}} & =\frac{\Delta \bar{W}_{\text {total }}}{2 T_{\mathrm{MMC}}}=\frac{H_{\mathrm{v}}}{T_{\mathrm{MMC}}} \Delta \bar{\omega}_{\mathrm{rec}} \\
& =\frac{-H_{\mathrm{v}} \bar{P}_{\mathrm{ac} 0} \bar{X}_{\Sigma} s}{T_{\mathrm{MMC}}\left(2 H_{\mathrm{v}} \bar{X}_{\Sigma} s^{2}+\bar{X}_{\Sigma} D_{\mathrm{v}} s+\omega_{0}\right)} \Delta \bar{U}_{\mathrm{g}}
\end{aligned}
$$

$P_{\mathrm{ac}}$ and $\omega_{\mathrm{g}}$ are considered as constants in Eq. (14). $P_{\mathrm{dc}}$ and $\omega_{\mathrm{g}}$ are considered as constants in Eq. (15). Both transfer functions in Eq. (14) and Eq. (15) have a high gain at around $100 \mathrm{rad} / \mathrm{s}$, which means that the DC voltage is sensitive to the change of $P_{\mathrm{dc}}$ and $U_{\mathrm{g}}$. When there is a disturbance, e.g., wind power fluctuation or grid voltage variation, the accuracy of grid frequency information delivered by DC voltage will be largely influenced, and may cause unexpected inertia response. In order to solve this problem, the decoupled DC voltage control of REC can be utilized.

\section{B. Decoupled DC Voltage Control of REC}

In the decoupled DC voltage control, the sum of the inserted submodules of the upper and lower arms is no longer a constant and considered as a controllable value $N_{\text {insert. }}$ The DC voltage generated by the SMs $E_{\mathrm{dc}}$ is:

$$
E_{\mathrm{dc}}=N_{\text {insert }} U_{\text {SMav }} \cdot
$$

The DC side equivalent circuit of the REC is shown in Fig. 2. $L_{\mathrm{eq}}$ is the equivalent inductance of the DC link inductance and $\mathrm{MMC}$ arm inductance. $0.5 C_{\text {line }}$ is the equivalent capacitance of the DC link capacitance. $U_{\mathrm{dc}}$ is the output DC voltage.

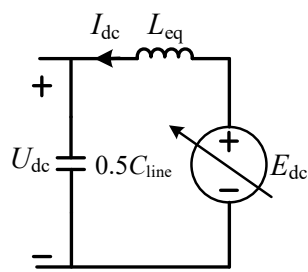

Fig. 2. DC side equivalent circuit of REC.

A closed-loop control structure is established in Fig. 3. By regulating $N_{\text {insert }}$ and $E_{\mathrm{dc}}, U_{\mathrm{dc}}$ can be controlled rapidly and precisely.

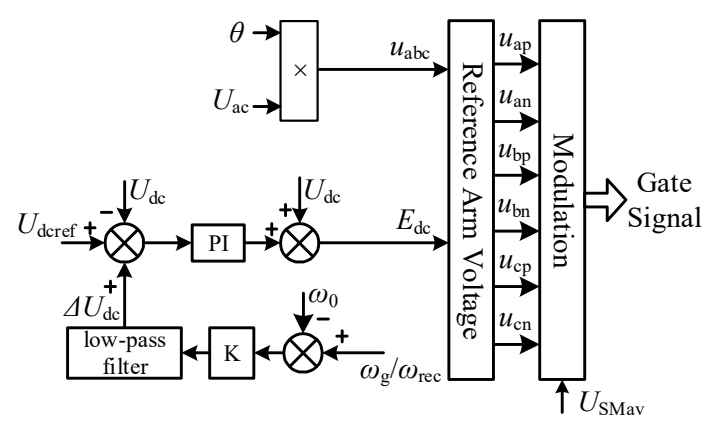

Fig. 3. Decoupled DC voltage control of REC.

In order to realize the communication-less inertia support from the SEC and the OWF, the grid frequency deviation $\omega_{\mathrm{g}}$ $\omega_{0}$ is converted to the change of the DC voltage reference $\Delta U_{\mathrm{dc}}$. The grid frequency $\omega_{\mathrm{g}}$ can be obtained from the local SCADA or the PLL. Also, according to Eq. (10), $\omega_{\text {rec }}$ can be applied here instead of $\omega_{\mathrm{g}}$. If $\omega_{\text {rec }}$ is applied here, a low-pass filter is required to filter out the disturbances mentioned in Eq. (14) and Eq. (15).

With the decoupled DC voltage control, the DC voltage is decoupled with the internal energy of the MMC, thus being robust to the disturbance of grid voltage variation and wind power fluctuation. And the accuracy of grid frequency information delivery will be improved.

\section{Overall CONTROl StruCture OF MMC-HVDC SYSTEM WITH WIND FARM INTEGRATION}

The overall control structure of the MMC-HVDC system and wind farms is shown in Fig. 4. For the REC, the total energy of SM capacitors $W_{\text {total }}$ is calculated and used for synchronization. The output $\mathrm{AC}$ voltage $U_{\mathrm{ac}}$ is utilized to control the reactive power $Q_{\mathrm{rec}}$ as well as provide damping term as is shown in Eq. (5). In order to decouple $U_{\mathrm{dc}}$ with $W_{\text {total }}$ and enhance the dynamic control performance of $U_{\mathrm{dc}}$, the decoupled DC voltage control method is applied.

As mentioned earlier, the REC is mainly responsible for achieving the autonomous grid synchronization while the process itself has limited inertia support to the grid. This issue can be resolved by coordinating the SEC control and the OWF. As shown in Fig. 4, when the SEC is informed with grid frequency variations by detecting the DC voltage, the grid frequency variation will be immediately reflected on the AC frequency of the wind farm.

$$
\Delta \bar{\omega}_{\mathrm{WF}}=\frac{1}{K} \Delta \bar{U}_{\mathrm{dc}} .
$$

For the wind turbines, an additional value associated with the rate-of-change-of-frequency (ROCOF) is attached to the active power reference $\left(P_{\mathrm{MPPT}}\right)$ given by the MPPT control. 


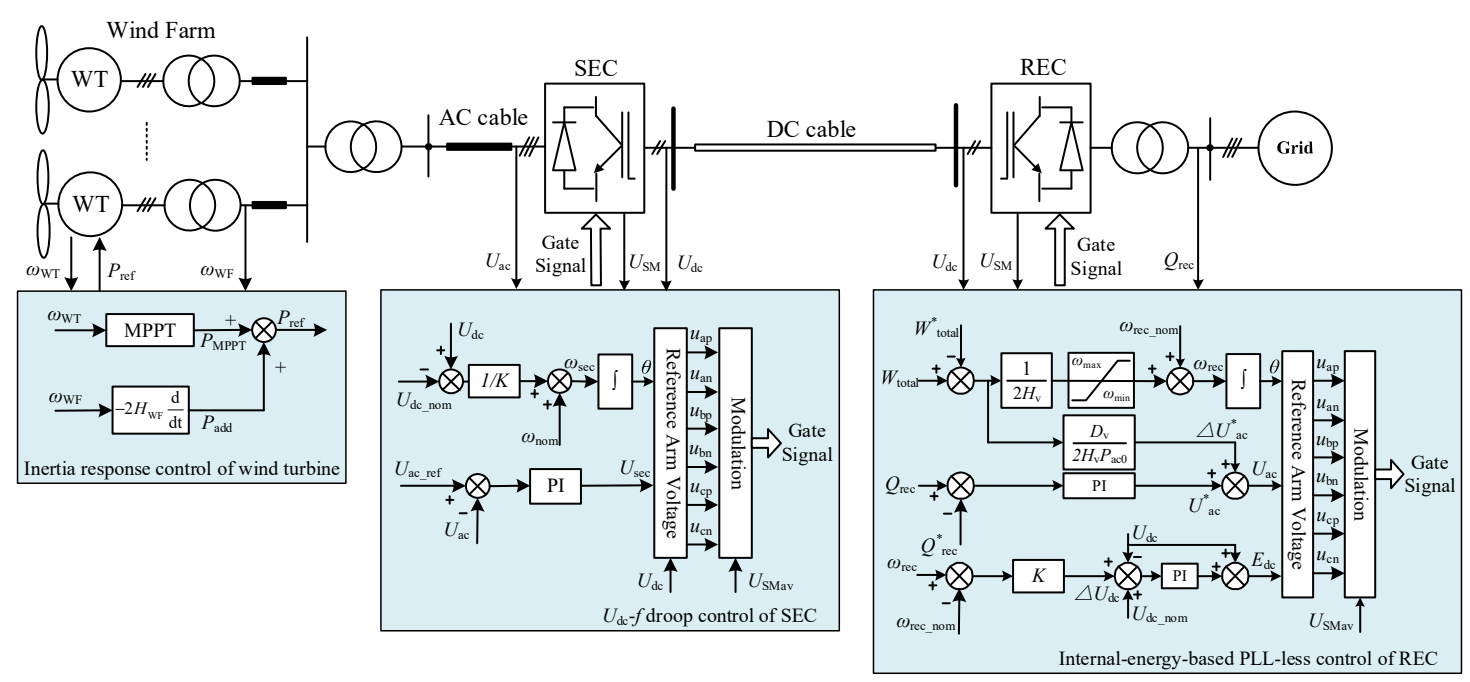

Fig. 4. Overall control diagram of REC.

The additional power $P_{\text {add }}$ is provided by accelerating or decelerating the wind turbine and utilizing the kinetic energy stored in rotating blades. Assuming that the virtual inertia of a wind farm is $H_{\mathrm{WF}}$, the value of $P_{\mathrm{add}}$ is:

$$
\bar{P}_{\mathrm{add}}=-2 H_{\mathrm{WF}} \frac{\mathrm{d} \bar{\omega}_{\mathrm{WF}}}{\mathrm{d} t}
$$

\section{Simulation Results}

In order to verify the effectiveness of the proposed control strategy, a simulation model is built in PSCAD/EMTDC. The model is based on the Rudong OWF MMC-HVDC system in China. The parameters of the HVDC system can be found in Table I. The AC grid is equivalent to a $10 \mathrm{GVA}$ SG and a 4GW load. The SCR is set to 2.5 , which represents a weak grid. The OWFs are aggregated to a 1000MW PMSG.

\section{Case1: Dynamic performance of the DC voltage control}

The output active power of the wind farm suddenly drops from 1 p.u. to 0.2 p.u. at $t=2 \mathrm{~s}$, and increase from 0.2 p.u. to $1 \mathrm{p}$.u. at $t=5 \mathrm{~s}$. The dynamic responses of the conventional DC voltage control and the decoupled DC voltage control are compared in this case. $H_{\mathrm{v}}$ and $D_{\mathrm{v}}$ of these control strategies are set to $0.05 \mathrm{~s}$ and 13 , respectively.

It can be observed from Fig. 5 that the DC voltage variation of the decoupled DC voltage control is $1.5 \%$, which is much smaller than the conventional DC voltage control. The simulation results prove that the decoupled DC voltage control method has excellent dynamic responses under sudden active power changes.

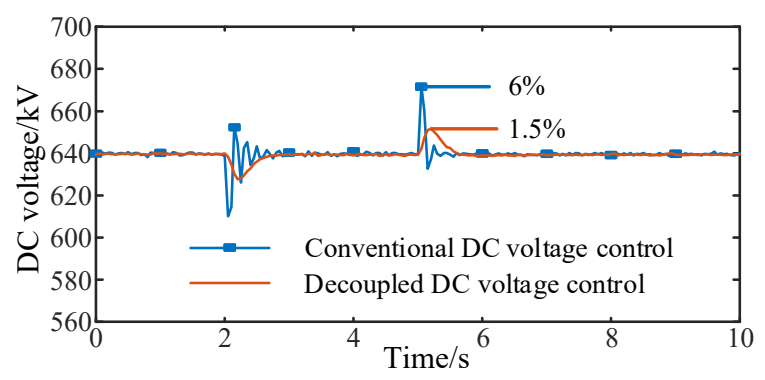

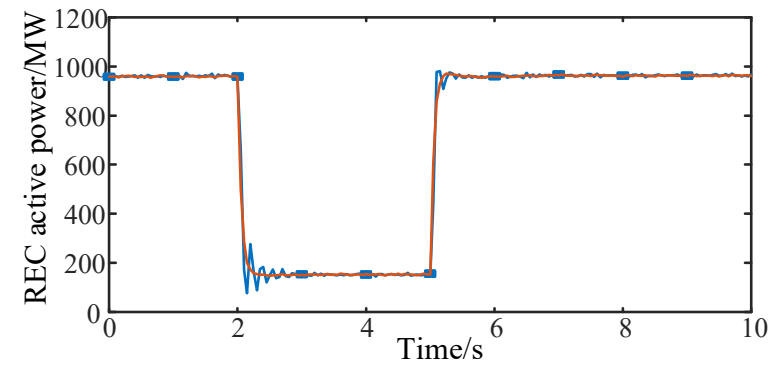

Fig. 5. Dynamic response of different control strategies in Case1.

\section{Case2: Inertia support}

Both decoupled DC voltage control and conventional DC voltage control are applied in this case. At $t=2 \mathrm{~s}$, the load increase from $4 \mathrm{GW}$ to $5 \mathrm{GW}$. It can be observed from Fig. 6 and Fig. 7 that, with both control strategies, the DC voltage tracks the grid frequency rapidly. With this feature, the wind farm can provide fast inertia support, i.e., approximately $10 \%$ of its rated active power can be delivered to the grid. Compared to those inertia-less control strategies, the ROCOF of the grid is reduced.

With conventional DC voltage control, it can be observed in Fig. 6 that at the beginning of inertia response, the DC voltage experience a transient dynamic. It is because that when the DC voltage tracks the grid frequency and decrease, the increase of active power from wind turbines will make DC voltage increase. This transient dynamic does not happen in Fig. 7, since the dynamic performance of DC voltage control with the decoupled DC voltage control is much better.

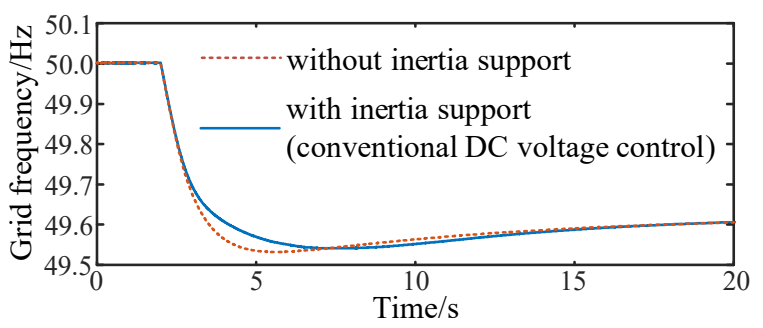



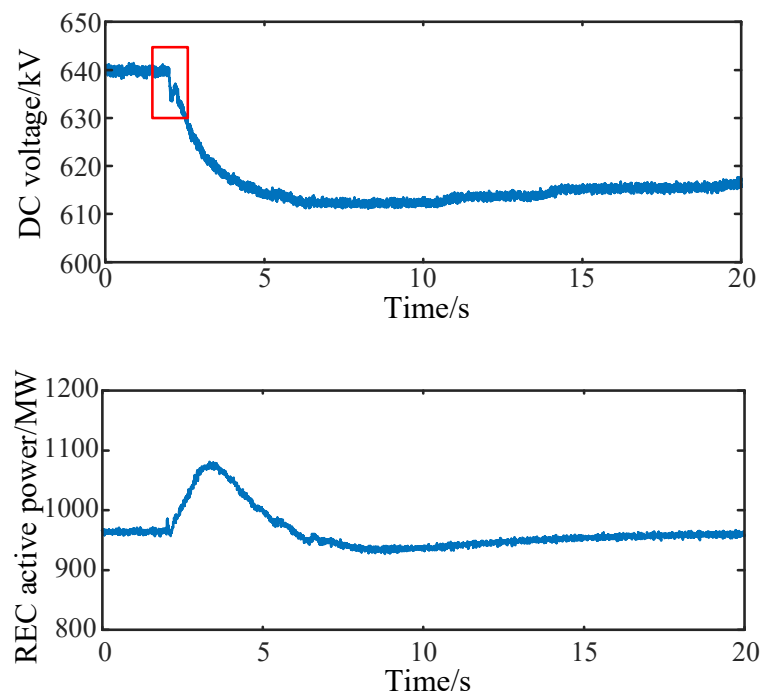

Fig. 6. Responses of the MMC-HVDC system and the wind farm with conventional DC voltage control in Case2.
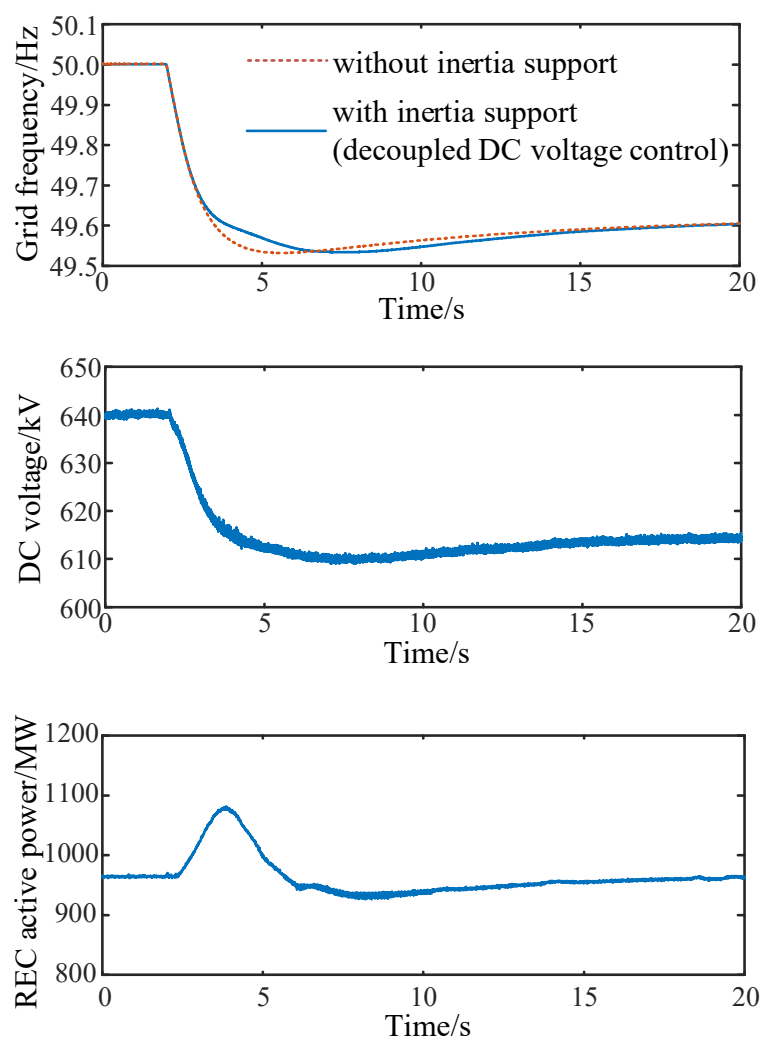

Fig. 7. Responses of the MMC-HVDC system and the wind farm with decoupled DC voltage control in Case2.

\section{CONCLUSION}

This letter presents an autonomous grid-synchronization control strategy using MMC's SM capacitor energy and applied to a practical test system of MMC-HVDC with OWF integration. Analysis has shown that the proposed method exhibits some superior performances in weak grid operation and particularly in achieving fast and autonomous inertia support compared to conventional control strategies.

The key control techniques developed enabling the above superiorities mainly lie in twofold: 1) the DC voltage control is decoupled from the SM capacitor voltage, which results in an excellent dynamic performance; 2) the grid frequency variation can be delivered to the wind turbines in an almost real-time manner, therefore, the synthetic inertia response from the wind farm can be delivered to the grid with a fewer time-delay.

\section{REFERENCES}

[1] G. Li, J. Liang, F. Ma, C. E. Ugalde-Loo and H. Liang, "Analysis of Single-Phase-to-Ground Faults at the Valve-Side of HB-MMCs in HVDC Systems," IEEE Trans. Ind. Electron., vol. 66, no. 3, pp. 24442453, March 2019.

[2] S. D'Arco and J. A. Suul, "Virtual synchronous machinesClassification of implementations and analysis of equivalence to droop controllers for microgrids," in Proc. IEEE Grenoble Conf., Jun. 2013, pp. 1-7.

[3] Q. Zhong and G. Weiss, "Synchronverters: Inverters That Mimic Synchronous Generators," IEEE Transactions on Industrial Electronics, vol. 58, no. 4, pp. 1259-1267, April 2011.

[4] L. Zhang, L. Harnefors, and H.-P. Nee, "Power-synchronization control of grid-connected voltage-source converters," in IEEE Transactions on Power System, vol. 25, no. 2, pp. 809-820, May 2010.

[5] W. Zhang, A. M. Cantarellas, J. Rocabert, A. Luna, and P. Rodriguez, "Synchronous power controller with flexible droop characteristics for renewable power generation systems," in IEEE Transactions on Sustainable Energy, vol. 7, no. 4, pp. 1572-1582, Oct. 2016.

[6] I. Cvetkovic, D. Boroyevich, R. Burgos, C. Li, M. Jaksic, and P. Mattavelli, "Modeling of a virtual synchronous machine-based gridinterface converter for renewable energy systems integration," in Proc. IEEE 15th Workshop Control Modeling Power Electron. (COMPEL), Santander, Spain, Jun. 2014, pp. 1-7.

[7] L. Huang et al., "A Virtual Synchronous Control for Voltage-Source Converters Utilizing Dynamics of DC-Link Capacitor to Realize SelfSynchronization," in IEEE Journal of Emerging and Selected Topics in Power Electronics, vol. 5, no. 4, pp. 1565-1577, Dec. 2017.

[8] R. Yang, C. Zhang, X. Cai and G. Shi, "Autonomous gridsynchronising control of VSC-HVDC with real-time frequency mirroring capability for wind farm integration," in IET Renewable Power Generation, vol. 12, no. 13, pp. 1572-1580, Sep. 2018.

[9] M. G. Taul, X. Wang, P. Davari and F. Blaabjerg, "Current Limiting Control With Enhanced Dynamics of Grid-Forming Converters During Fault Conditions," in IEEE Journal of Emerging and Selected Topics in Power Electronics, vol. 8, no. 2, pp. 1062-1073, June 2020.

[10] W. Yang, Q. Song and W. Liu, "Decoupled Control of Modular Multilevel Converter Based on Intermediate Controllable Voltages," in IEEE Transactions on Industrial Electronics, vol. 63, no. 8, pp. 46954706, Aug. 2016. 